\title{
Optical Imaging Of Fast, Dynamic Neurophysiological
}

\section{Function}

David M. Rector, Kathleen M. Carter, Xincheng Yao, and John S. George

Los Alamos National Laboratory, Biophysics Group, MS-D454, Los Alamos, NM, 87545

e-mail: drector@lanl.gov phone:505-665-6230

BIOMED OSA Topical Meeting April 7-10, 2002

OSA Workshop Session: Direct Optical Measurements of Neuronal Signals

\section{Abstract:}

Fast evoked responses were imaged from rat dorsal medulla and whisker barrel cortex. To investigate the biophysical mechanisms involved, fast optical responses associated with isolated crustacean nerve stimulation were recorded using birefringence and scattered light. Such studies allow optimization of non-invasive imaging techniques being developed for use in humans.

\section{Summary:}

A number of studies utilized bright field illumination of neural tissue to image intrinsic absorbance changes associated with slow hemodynamic responses. These methods produce moderate-resolution spatial activation maps from the cortical surface with slow time resolution (e.g. Malonek and Grinvald, 1997; Narayan, et al., 1994; Polley et al., 1999; others). In order to capture dynamic spatio-temporal patterns, other investigators have employed voltage sensitive dyes (e.g. Shoham et al. 1999; Wackowiak and Cohen, 2000; others) at the expense of more invasive procedures. One of our ultimate goals is to use non- or minimally-invasive optical procedures to image fast, dynamic neurophysiological function closely correlated to electrical activation with high spatial and temporal resolution. The primary obstacle in these measurements is the sensitivity required to detect small optical changes on a large background. We have been developing imaging techniques to measure fast optical changes that follow electrical activity using a variety of paradigms including birefringence and dark-field illumination.

Over the past 60 years, investigators have recorded fast optical changes that track electrical events within various types of isolated nerve preparations. However, the measurement of fast dynamic optical signals has been difficult to achieve from intact neural tissue or in-vivo. Our early experiments on cat hippocampus used sensitive photodiodes to measure an integrated response to electrical stimulation of the crossed Schaeffer's collaterals over a large portion of tissue (Rector et al., 1997). At the time, our imaging technology was too slow to track the electrical responses of the hippocampus. During these experiments, it became clear that fast optical changes which track electrical events could be optimized by dark-field recording techniques (Stepnoski et al., 1991; Holthoff and Witte, 1996).

The use of fiber optics in reflectance imaging greatly increases the detection sensitivity for light scattering changes over lens coupled devices. Reflectance studies which use a lens coupled system typically illuminate the tissue surface and detect at a distance. High light levels are required, and most of the illumination detected by the camera is specular reflection from the tissue surface or scattering from superficial tissue. Only a small proportion of the light detected in this configuration interacts with tissue beneath the surface of the cortex. To detect such small changes, Grinvald and colleagues (1986) and most other investigators utilized large, high sensitivity, cooled camera equipment with slow scan rates.

We developed another method of detecting scattered light using fiber optic image conduit or a gradient index lens. The image conduit is in direct contact with both the tissue and the camera. Light is delivered to the tissue around the perimeter of the probe as in dark-field illumination. Thus, before entering the image conduit fibers, light below the tissue surface. Because of improved collection efficiency $=$ is technique does not require high illumination levels, miniature LEDs are adequate. The geometry of light paths from illumination fibers to detection fibers is such that a large portion of scattered light is perturbed by 
cells which change their scattering properties with activation. Such procedures greatly enhance the contrast of tissue activity changes over reflection mode imaging (Rector et al., 1999).

Using a novel imaging probe, we imaged fast intrinsic optical responses to vagal nerve stimulation within the nucleus tractus solitarius (NTS) of the rat dorsal brainstem. Dark field illumination enhanced the contrast of light scattering changes, that track electrical dynamics of neuronal activation. Vagal nerve stimulation elicited characteristic spatial and temporal optical patterns within the rat brainstem, corresponding to at least four distinct physical processes. Subregions within the image disclosed distinct spatial patterns associated with four temporal waveforms within the response: an early reflectance increase (P30) that corresponded temporally with the population action potential, an intermediate reflectance decrease (N80) that paralleled the population post-synaptic potential, a late and long-lasting reflectance decrease (N300) that may represent an early tissue deoxygenation, and a slow reflectance increase (P800) that corresponds to an over-compensatory influx of oxygenated blood to the activated tissue. Schematic versions of the four temporal response components served as basis functions in a weighted linear analysis. We modeled an early negative going response (P30) and an intermediate positive response (N80) after electrical signals. A late long lasting positive response (N300) and a slow negative going response (P800) were more widespread and were designed to be consistent with hemodynamic responses. Principal component analysis of the image sequence reveals two strong components that resemble the $\mathrm{N} 80$ and $\mathrm{N} 300$ waveforms, with similar spatial distributions. The first component (C1) parallels the N80 response and the second component (C2) parallels the N300 response and contains image patterns that overlap with blood vessels. Basis function reconstruction accounted for $91.5 \%$ of the variance (Rector et al., 2001).

The faster two responses appear to represent direct neural response components not seen in previous imaging studies. Such fast responses may be attributed to a number of biochemical or cellular processes that could change light scattering properties of neural tissue with activation. Neural swelling accompanies activation, with time courses which can parallel the action potential. The early response (P30) appears correlated with presynaptic activity in the terminal projections of the vagus. There is some evidence from this work and others (Salzberg and Obaid, 1985) that the optical signal precedes the electrical signal, possibly because neural swelling at the stimulus site initiates a compression wave which may travel near the speed of sound. Differences were also observed across depth for optical signals associated with vagal stimulation: the N80 fraction of the response increased in amplitude and the N300 fraction decreased in amplitude in deeper tissue layers across all animals recorded $(p<.01)$.

We also imaged the rat whisker barrel cortex during whisker deflection and observed fast scattered light changes that followed the electrical evoked response. Images revealed localized regions that responded to single whisker deflections. Stimulation of different whiskers moved the region of activation according to the expected anatomical mapping of the barrel field.

To investigate the biophysical mechanisms of the fastest optical components, we measured optical changes associated with stimulation of crustacean nerves using a combination of birefringence and 90 degree scattered light. Previous experiments with isolated crustacean nerves were confounded by multiple superimposed action potentials which hindered discrimination of optical temporal signatures (Cohen, 1973; Tasaki and Byrne, 1992). We utilized high speed photodiodes and amplification circuitry to achieve high time resolution, and observed multiple peaks in the optical signal appearing at different points in time during the compound action potential, apparently representing different axonal populations. Optical responses associated with large population action potentials from both crayfish and lobster nerve bundles show fast and slow temporal signatures with slight differences between species and type of nerve. Crayfish claw nerves typically produced optical responses that were $25 \%$ of the largest lobster leg nerve responses. A small aluminized mylar plate placed on the lobster leg nerve bundle surface and used as an optical lever showed a $10 \mathrm{~nm}$ displacement of the bundle.

We used a $1 \mathrm{~mm}$ pinhole to reduce the temporal integration of the birefringence signal. 
When the pinhole was placed over the nerve, the optical signal showed greater temporal structure and was delayed when compared to the optical signal integrated over the whole nerve. Moving the slit $3 \mathrm{~mm}$ closer to the stimulation wires moved the optical response $3 \mathrm{~ms}$ ahead in time and provided an enhanced early component.

Observation of simultaneously recorded birefringence and scattered light revealed differences in temporal structure of the signals. A fast component in the birefringence signal appeared early during electrical activation and a larger slower response persisted for several milliseconds afterward. The $90^{\circ}$ scattered light change lagged behind the birefringence by $1 \mathrm{~ms}$ and lacked some of the resolution that was seen in the birefringence signal primarily due to its smaller amplitude ( $10 \%$ of the birefringence signal).

Our image probe can be configured to create confocal, spectrally resolved images of the tissue for better spatial resolution, better biochemical and physiological specificity, and allow 3$D$ reconstruction of the tissue activation volume. High-speed and high-sensitivity optical recordings are minimally invasive and offer unprecedented spatial and temporal resolution. These methods may allow detailed studies of dynamic temporal and spatial activity patterns underlying high-level processing within local neural networks that perform sensory, cognitive and autonomic functions.

Such studies will allow us to optimize noninvasive imaging techniques being developed for use in humans (Haglund et al, 1992; Villringer and Chance, 1997; Gratton et al., 1997; Steinbrink et al., 2000;). Noninvasive optical measures could increase the effectiveness of existing techniques, such as EEG and functional MRI by resolving both the fast neuronal responses and slower vascular responses. This method could also lead to fast, cost effective, and noninvasive techniques for pathology and monitoring associated with stroke or head trauma because of its ability to detect intrinsic light changes in many different types of molecules.

\section{References:}

Cohen, L.B. Changes in neuron structure during action potential propagation and synaptic transmission. Physiol. Rev., 53(2):373-413, 1973.

Gratton, G., Fabiani, M., Corballis, P.M., and Gratton, E. Noninvasive detection of fast signals from the cortex using frequency-domain optical methods. Ann. N.Y. Acad. Sci., 820:286-99, 1997.

Grinvald, A., Lieke, E., Frostig, R.D., Gilbert, C.D., and Wiesel, T.N. Functional architecture of the cortex revealed by optical imaging of intrinsic signals. Nature, 324:361-364, 1986.

Haglund, M.M., Ojemann, G.A., and Hochman, D.W. Optical imaging of epileptiform and functional activity in human cerebral cortex. Nature, 358:668-671, 1992.

Holthoff, K., Witte, O.W. Intrinsic optical signals in rat neocortical slices measured with near-infrared dark-field microscopy reveal changes in extracellular space. J. Neurosci., 16(8):2740-2749, 1996.

Malonek, D. and Grinvald, A. Vascular regulation at sub millimeter range. Sources of intrinsic signals for high resolution optical imaging. Adv. Exp. Med. Biol, 413:215-220, 1997.

Narayan, S.M., Santori, E.M., and Toga, A.W. Mapping functional activity in rodent cortex using optical intrinsic signals. Cereb. Cort., 4(2): 195-204, 1994.

Polley D.B., Chen-Bee C.H., Frostig R.D. Varying the degree of single-whisker stimulation differentially affects phases of intrinsic signals in rat barrel cortex. J. Neurophysiol., 81(2):692-701 1999.

Rector, D.M., Poe, G.R., Kristensen, M.P., and Harper, R.M. Light scattering changes follow evoked potentials from hippocampal schaeffer collateral stimulation. J. Neurophysiol., 78:1707-1713, 1997.

Rector, D.M., Rogers, R.F., and George, J.S. A focusing image probe for assessing neural activity in-vivo. J. Neurosci. Meth., 91:135-145, 1999.

Rector, D.M., Rogers, R.F., Schwaber, J.S., Harper, R.M. and George, J.S., Scattered Light Imaging In-Vivo Tracks Fast and Slow Processes of Neurophysiological Activation. Neurolmage, 14, 977-994, 2001.

Salzberg, B.M., Obaid, A.L., and Gainer, H. Large and rapid changes in light scattering accompany secretion by nerve terminals in the mammalian neurohypophysis. J. Gen. Physiol., 86:395-411, 1985.

Shoham D., Glaser D.E., Arieli A., Kenet T., Wijnbergen C., Toledo Y., Hildesheim R., Grinvald A. Imaging cortical dynamics at high spatial and temporal resolution with novel blue voltage-sensitive dyes. Neuron. 24(4):791-802, 1999.

Steinbrink, J., Kohl, M., Obrig, H., Curio, G., Syre, F., Thomas, F., Wabnitz, H., Rinneberg, H., Villringer, A. Somatosensory evoked fast optical intensity changes detected non-invasively in the adult human head. Neurosci. Lett., 291(2): 105-8, 2000. 
Stepnoski R.A., LaPorta, A., Raccuia-Behling, F., Blonder G.E., Slusher R.E. and Kleinfeld D. Noninvasive Detection of Changes in Membrane Potential in Cultured Neurons by Light Scattering. PNASUSA, 88(21):9382-9386, 1991 .

Tasaki, I. and Byrne, P.M. Rapid structural changes in nerve fibers evoked by electrical current pulses. Biochem. and Biophys. Res. Comm., 188(2):559-64, 1992.

Wachowiak M., Cohen L.B. Representation of odorants by receptor neuron input to the mouse olfactory bulb. Neuron, 32(4):723-735, 2001.

Villringer A., Chance B. Non-invasive optical spectroscopy and imaging of human brain function. TINS, v. 20(10):435-442, 1997. 